\title{
Quaderni
}

QUADERNI Communication, technologies, pouvoir

75 | Printemps 2011

Le Parlement face aux TIC

\section{Entretien de Lucien Sfez avec Daniel Dayan}

\section{Daniel Dayan et Lucien Sfez}

\section{(2) OpenEdition \\ Journals}

\section{Édition électronique}

URL : http://journals.openedition.org/quaderni/405

DOI : 10.4000/quaderni.405

ISSN : 2105-2956

\section{Éditeur}

Les éditions de la Maison des sciences de l'Homme

\section{Édition imprimée}

Date de publication : 5 avril 2011

Pagination : 73-84

Référence électronique

Daniel Dayan et Lucien Sfez, « Entretien de Lucien Sfez avec Daniel Dayan », Quaderni [En ligne], 75 | Printemps 2011, mis en ligne le 05 avril 2013, consulté le 01 mai 2019. URL : http:// journals.openedition.org/quaderni/405; DOI : 10.4000/quaderni.405 


\section{Entretien}

\section{entretien de questrons sur daniel dayan}

\section{Lucien Sfez Lucien S Ster: Comment vous deffinirier vous?}

Daniel Dayan : Je suis un sociologue de l'image et je m'intéresse en particulier à celles que proposent les médias et le journalisme. Mon

Daniel Dayan

Daniel

Dayan

Directeur de Recherche émérite au CNRS

Lucien

Sfez

Professeur émérite Université de Paris 1 Panthéon Sorbonne

Daniel Dayan a reçu le "Fellows Award" pour le livre qu'il a signé avec Elihu Katz intitulé Media events et traduit en français sous le titre

La Télévision cérémonielle, paru aux PUF.

Ce prix est décerné pour couronner un ouvrage classique par l'International Communication Association originalité par rapport à d'autres sociologues des médias est d'être parti de l'esthétique, de la théorie du cinéma, puis de l'étude d'une forme de télévision longtemps marginale par rapport à la majorité des émissions télévisuelles : la télévision cérémonielle. Comme le «drame baroque allemand» étudié par Benjamin, ce genre médiatique est important non seulement pour lui même, mais pour l'éclairage qu'il offre sur l'ensemble du paysage télévisuel et journalistique.

\section{S. : Pouvez vous résumer vos principales} thèses en deux ou trois points?

D.D. : J'aimerais combiner ici deux des thèmes que j'ai abordés (1) la critique du journalisme (2) en référence à l'analyse des événements « expressifs ».

Commençons par le premier. Sans renoncer à se présenter comme des « observateurs » du monde politique, et du monde en général, un certain nombre de grands médias en sont devenus les acteurs essentiels, décidant de ce qui est visible ou pas ; de ce qui fait problème ou pas ; du moment où une question donnée dominera l'espace public ; décidant si elle le fera longtemps ou pas, si elle le fera exclusivement ou pas. Devant ce rôle souverain qui consiste à dire ou à taire 
le politique, devant cette gestion de l'attention collective, les acteurs politiques se trouvent relégués à des seconds rôles. Il leur en reste deux.

Le premier relève d'une une sorte de ventriloquie. Qu'un média veuille traiter un thème: il se trouvera toujours un homme politique pour lui prêter sa voix et ses lèvres. Comme naguère les 'experts' (qui étaient toujours les experts de quelqu'un), les hommes politiques sont devenus les porte-voix plus ou moins volontaires d'un discours dont les véritables auteurs sont les grands médias.

Le second de ces rôles est un rôle de réaction. Voici - disent les médias - ce à quoi il faut que vous réagissiez. «Eh bien, réagissez maintenant!». Les acteurs politiques s'exécutent. Dans une sphère publique de la visibilité, la réaction est obligatoire puisque le fait de ne pas réagir sera lui-même comptabilisé comme une réaction. Les acteurs politiques identifiés comme tels ont alors le choix entre deux rôles mineurs : servir de marionnettes à un ventriloque ; servir de « réagissants » à des figures imposées.

Cette constatation entraîne plusieurs conséquences pour l'analyse du journalisme et notamment celle-ci. Le journalisme consiste non plus seulement à décrire une réalité mais à la constituer. Les journalistes contemporains ont le privilège de décider par leur discours de ce qu'est la réalité sociale ou de ce qu'a été celle des événements. En un mot, le journalisme ne relève pas du constat mais de la performance.

L.S. : Tenez-vous compte ici de la survenue inopinée d'événements? Une révolution, un accident d'avion, une épidémie ne sont pas contrôlés par les médias. Ils sont ou ne sont pas. Comment alors faire entrer l'information dans votre logique?

D.D. : Pour répondre à votre question je propose de distinguer comme le fait Paddy Scannell, deux sortes d'événements tous deux présents dans les nouvelles : les événements « expressifs » et les événements « happenings ».

Les événements expressifs ne sont pas véritablement des événements. Ce sont des événements voulus. Ils sont non pas ce qui arrive, mais ce qu'on fait arriver afin d'illustrer un propos ou de célébrer une valeur. Boorstin parlerait à ce propos de " pseudo-événements ». Les médias contemporains sont parfaitement à l'aise avec ce genre d'événements qu'ils maîtrisent. Certains de ces événements sont consensuels. Ce sont ceux que j'analyse dans la Télévision cérémonielle. D'autres sont délibérément dissensuels, agonistiques, comme par exemple l'organisation d'une flottille partant en grande pompe briser le blocus de Gaza, ou celle d'un autobus de rock stars quittant Londres pour Bagdad à la veille de la guerre pour dénoncer l'invasion américaine de l'Irak. De tels événements ne sauraient exister sans une co-production avec les médias. (Le bateau Mavi Marmara est un véritable studio de télévision flottant pour la télévision turque et Al Jazira). Les événements expressifs offerts dans les nouvelles participent donc à l'entreprise de construction médiatique de la réalité.

Par contre, il existe des événements « happenings ». Ce sont de véritables événements en ce 
sens qu'ils sont ce qui arrive, qu'on le prévoie ou pas, qu'on le veuille ou pas : tsunamis, révolutions, chutes de régimes. Souvent leur caractère irruptif rend de tels événements difficilement racontables à partir du stock des récits disponibles ou consacrés. Pour raconter un événement nouveau, il faut souvent faire appel à un récit qui le soit aussi. Ceci peut plonger les médias dans l'embarras. Trois possibilités se présentent alors. (1) Mettre en place des récits inédits. Cela peut arriver, mais pas si souvent. (2) Ignorer l'événement nouveau, faute de savoir ou de vouloir le raconter. Ainsi Edwy Plenel avoue t-il avec un certain embarras que Le Monde néglige pendant de longs mois de parler du Rwanda parce que, vu depuis Paris, le génocide en cours semble n'être qu'un conflit africain de plus. (3) Raconter l'événement nouveau à l'aide d'un récit consacré, même si ce dernier se révèle grossièrement inadéquat. Je suis ainsi frappé par le nombre de fois où un événement radicalement nouveau est non pas ignoré, mais simplement remplacé par un autre événement plus digestible, ou par une controverse livrée avec son stock d'arguments prêts-à-parler, et sa ménagerie d'acteurs politiques prêts à passer à l'antenne.

Par exemple l'attaque meurtrière contre la gare d'Atocha à Madrid est remplacée par une interrogation qui serait raisonnable si elle ne finissait pas par faire oublier l'événement luimême. José Maria Aznar mentait-il quand il évoquaitlapossibilitéd'uneimplicationbasque ?

Pour emprunter une distinction à Lévi-Strauss on pourrait dire que les grands médias n'aiment pas les événements happenings « crus ». Ils ne les aiment que quand ils les ont « cuits ». Une fois cuits, les « événements-happenings » sont dotés d'un sens, d'une signification, d'une postérité. Ils deviennent donc à leur tour des " événements-expressifs », des événements compatibles avec une certaine construction de la réalité.

\section{L.S. : Vous semblez opposer non seulement les informations à l'événement véritable, mais les informations à la notion même d'information.}

D.D. : Notre espace public est un espace de réactivité. Le modèle pourrait en être celui du pavé dans la mare et des ondes qu'il provoque. Il est normal que le système médiatique s'intéresse aux ondes provoquées par le pavé. Il est beaucoup moins normal que le système médiatique se désintéresse si souvent du pavé lui-même.

\section{L.S. : La machine à informations pourrait alors très bien fonctionner sans événements du tout? N'est-ce pas paradoxal?}

D.D. : Oui c'est paradoxal, mais le phénomène n'a rien de nouveau. C'est à un phénomène de ce type que pensait Hegel lorsqu'il comparait la lecture quotidienne du journal à une messe dont les fidèles seraient dispersés. Et, il y a près de trente ans, dans un livre désormais classique sur la BBC (Putting 'reality'together), Philip Schlesinger montrait que, dans leur grande majorité, les images utilisées dans la plupart des bulletins de nouvelles étaient mises en boîte plusieurs jours à l'avance. Un événement véritable malmène les grands récits 
et les croyances qui les sous-tendent. De plus, rapporter l'événement coûte cher. Ainsi devientil plus économique pour Libération de ne plus faire d'information du tout, d'abandonner les « pavés», de se spécialiser dans les « ondes» et les « rebonds », de devenir un magazine-auquotidien.

\section{L.S. : Quelles conséquences tirez-vous de tout ceci ?}

D.D. : Je dirais - comme J. B. Thompson qu'il n'existe plus de réalité politique qui soit totalement immédiate (non médiatique). Une réalité non médiatique devient simplement une réalité en attente de le devenir. Dans une sphère publique de la monstration, voire de la signalisation, il n'y a plus de non-médiatique. Il subsiste seulement du pré-médiatique.

L.S. : Les journalistes ont été incapables de prévoir les révolutions égyptienne et tunisienne. Ils ont été suivistes face à de tels événements. Ceci ne limite-t-il pas sérieusement leur pouvoir de construire la réalité ? $\mathbf{N}^{\prime}$ y a-t-il pas des situations où la réalité sociale se manifeste?

DD Certes les événements font irruption dans nos espaces publics. Mais combien de jours ont-ils pour faire leurs preuves avant d'être enfermés dans le carcan d'un récit ? Et peuvent-ils échapper à un tel carcan ? Les révolutions égyptienne ou tunisienne ont deux possibilités. Servir d'étendards à de grands récits de libération - ou - disparaître de l'espace public. Une narration manichéenne est là, prête à s'emparer des événements. Que se passe-t-il alors si, au lieu d'illustrer un récit épique, ces événements relèvent plutôt du récit nuancé d'un Orhan Pamuk ? Que se passe-t-il lorsqu'il n'y a pas de bataille entre un bien et un mal, mais une situation ambiguë ? Lorsque d'un côté on trouve un progressisme autoritaire et imposé par des militaires ? Lorsque de l'autre on trouve, des aspirations démocratiques qui mènent au fondamentalisme religieux ? Comment raconter une histoire où le progressisme et la démocratie s'affrontent? Il est sans doute plus facile de ne pas la raconter du tout. Pourquoi alors ne pas offrir, à la place, un récit circonstancié des pérégrinations aériennes de Madame Alliot-Marie?

\section{LES INFLUENCES : \\ BARTHES, GOFFMAN, AUSTIN}

\section{L.S. : Quels sont vos trois auteurs-phares ?}

D.D. : Mes trois auteurs-phares se trouvent chacun à la limite de plusieurs disciplines. Tous trois font une place à la linguistique, et tous trois ont un rapport avec le théâtre. Tous trois manifestent enfin un côté ludique qui les fait volontiers passer pour des « amateurs ». L'un a été mon directeur de thèse. Le second a failli l'être. Je n'ai jamais rencontré le troisième.

Commençons par mon mentor effectif, Roland Barthes. Barthes était critique littéraire, théoricien de la narrativité, et comme il le disait « sémiologue » plutôt que sémioticien. En d'autres termes, la technicité sémiotique ne l'intéressait pas. Ce qu'il appelait « sémiologie » était une fidélité à son propre mentor, Bertolt Brecht. La linguistique permettait une sorte de mise-en-crise brechtienne de la culture. 
Lorsque j'ai été son élève, Barthes enseignait la « sociologie des signes, symboles et significations ». Pour lui il s'agissait d'un pavillon de complaisance (il lui permettait d'enseigner à l'EHESS ). Pour moi c'était une révélation. À partir de L'écriture de l'événement, Le discours de l'Histoire, Mythologies, j'ai appris de Barthes que les réalités sociales les plus lourdes sont le plus souvent des signes et que les réalités apparemment les plus futiles peuvent être cruciales. J'ai appris que l'on peut le mieux se livrer à une activité de déchiffrement du social à partir d'exemples délibérément choisis comme petits. J'ai appris à regarder lentement, avec intensité et en détail et à ne jamais sacrifier ce détail à une volonté surplombante de synthèse.

\section{L.S. : Mais en quoi sa pensée a-t-elle pu enrichir vos analyses?}

D.D.: J'ai appris de Barthes qui était la politesse incarnée cette profonde impolitesse qui consiste à analyser des situations sérieuses ou tragiques comme si elles étaient futiles, car cette «futilité" révèle souvent ce en quoi elles sont spécifiques. Par exemple, j’ai appris de Barthes à lire ce qu'on a appelé « les événements de Mai » comme une chorégraphie réglée, une sorte de pas de deux mobilisant la rive gauche et la rive droite selon un scénario dont l'ambition était de signifier la révolution mais pas vraiment de la faire advenir. Cette lecture «formaliste» m'a énormément appris, par exemple, sur le terrorisme. Elle m'a permis de concevoir le terrorisme comme un ensemble de dramaturgies publiques visant à signifier la guerre à l'aide de violences généralement réduites au minimum. Je me suis intéressé à chacune de ces dramaturgies en me demandant par exemple qui enregistrait les kamikazes à la veille de leurs missions meurtrières ou pourquoi les assassins de Daniel Pearl s'étaient faits filmer avec des fusils d'assaut, alors que l'exécution du journalisme prendrait la forme d'un égorgement à l'arme blanche. C'est un détail ? Je ne crois pas. Le recours à l'égorgement nie l'humanité de l'égorgé. La voix d'«outre-tombe» du kamikaze confère une dimension de proximité romanesque avec celui-ci. À partir de tels détails on peut anticiper l'évolution des dramaturgies, et donc prévoir l'émergence de nouvelles formes qui remplaceront le terrorisme

À l'inverse, il me semble que l'absence d'une attention barthienne portée au détail nuit gravement à toutes sortes d'analyses. Prenons un autre exemple analysé par S. Boudana, celui d'un scandale estival qui a fait le tour du monde : la noyade sur une plage italienne de deux fillettes tziganes. La photo des deux petits cadavres enveloppés dans des serviettes de bain et laissés sur la plage au milieu de vacanciers indifférents accuse l'Italie berlusconienne, témoigne de sa cruelle xénophobie. Cette xénophobie existe, mais il me semble important d'entrer dans le détail. Pourquoi sur les nombreuses photos prises par le photographe la seule retenue est celle dont la focale masque la distance entre les baigneurs et les deux corps ? Pourquoi n'a t-on pas précisé que la majorité de ces vacanciers ne sont pas italiens? Pourquoi n'a-t-on pas dit que les tapis de bain qui servent de linceuls, sont ceux que des vacanciers ont sacrifiés par compassion pour les fillettes? Pourquoi enfin n'a-t-on pas dit que ce jour là, il y avait non pas 
deux fillettes mais quatre fillettes en train de se noyer, et que si deux seulement sont mortes, c'est parce que des vacanciers «cyniques» et « racistes » se sont jetés à l'eau pour sauver les deux autres? Qu'une seule situation puisse donner lieu à une telle cascade d'approximations, d'erreurs, d'omissions et de mensonges en dit long sur le rôle que se donne ici le journalisme. Hélas, comme l'a montré Errol Morris, de telles situations n'ont rien d'exceptionnel. S'agit-il alors de rapporter un événement ou de conforter des « Mythologies ». Et si l'événement contredit le mythe, lequel des deux, à votre avis, va-t-on remanier? J'ai appris de Barthes à lire le récit des nouvelles comme un texte littéraire ou pictural parmi d'autres, un peu plus grossier que d'autres. Malgré des idéaux documentaires affichés, la dénotation y devient un alibi permanent pour les connotations désirées.

\section{L.S. : Quel est votre second auteur-phare ?}

D.D. : C'est Erving Goffman. Tout comme Barthes, Goffman a réussi à se faire passer pour un sociologue. Il enseignait la sociologie à l'Université de Pennsylvanie (où il m'a offert une bourse en I965). Il a même été président de l'American Sociological Association. Quand on le lit, on a pourtant l'impression que Goffman n'est pas vraiment sociologue. Peut être estil linguiste? psychologue? Longtemps avant Latour, il réussit à se passer de la société (avec un grand S) et propose plutôt une « associologie », s'intéresse aux formes de la sociabilité.

\section{L.S. : Même question que pour Barthes. En quoi la pensée de Goffman a-t-elle pu enrichir vos analyses?}

D.D. : J'ai été influencé par la cohérence de la vision goffmannienne d'une société abordée comme une architecture d'interactions. De cette œuvre protéiforme et néanmoins rigoureusement cohérente trois thèmes m'ont particulièrement influencé.

Chacun de nous procède à de constantes «présentations de soi » dans la vie de tous les jours. Nous passons notre temps à décliner notre identité, et, comme le suggère le mot « décliner», nos identités. Mon travail sur la « télévision cérémonielle » consistait à explorer certains moments où ce sont des sociétés tout entières qui éprouvent le besoin de procéder collectivement à des «présentations de soi »dans des circonstances exceptionnelles et souvent ritualisées. Le thème goffmanien de la présentation-de-soi me permettait alors de décrire une nouvelle forme de «religion civile»; une religion où des "présentations de soi » ad hoc viendraient se substituer aux cérémonies prévues par les calendriers festifs ; une religion dont les rituels serviraient parfois à confirmer des identités collectives, parfois, au contraire à les redéfinir ou à les reformuler.

Un second aspect de la pensée de Goffman continue à m'inspirer, presque quotidiennement. Goffman parle du droit - crucial pour tout individu libre, c'est-à-dire, ni prisonnier, ni interné - à maintenir une distinction entre une « région façade » où chacun décide s'il veut se montrer et sous quelle forme et une « régionrefuge » inaccessible à la visibilité. De façon intéressante, me semble-t-il, Goffman qui s'intéresse aux prisons, aux hôpitaux, aux asiles, aborde à peu près en même temps que Foucault 
l'un des grands thèmes du philosophe français. Pour Foucault, le passage d'une société de la « punition » à une société de la « surveillance» institue en effet une situation où le sujet est constamment exposé au regard d'autrui. Foucault utilise une métaphore architecturale (le fameux «panopticon »). Goffman préfère une métaphore dramaturgique (l'absence de coulisse). Plus peut-être que la référence au " panopticon », l'organisation de la vie sociale en activités de scène et de coulisses permet de réfléchir sur un phénomène comme Wikileaks. Elle m'amène par exemple à me demander ce que serait un espace public où tout serait sur scène et où il n' y aurait plus de coulisses. Pour nombre d'observateurs, cet espace représenterait un idéal qui s'incarnerait dans le journalisme d'investigation, ou dans ce que j'appellerais de façon plus juste un journalisme de «dévoilement». S'agit-il d'un idéal ou d'un cauchemar ? Peut-on vivre sans « région refuge $»$ ?

Ma troisième dette envers Goffman est la suivante. Dans toute situation de parole, Goffman distingue entre plusieurs rôles du locuteur. Celui-ci peut être (1) l'auteur du discours tenu (celui qui l'a conçu, et mis en mots ), (2) le responsable du discours tenu (celui qui serait passible de poursuites si ce discours contrevenait à la loi ). Il peut enfin devenir (3) l'animateur du discours tenu (celui qui prononce les mots, leur prête son corps, ses lèvres, sa voix, ses expressions, comme s'il était, par exemple, un acteur). Dans la vie courante nous sommes généralement les trois à la fois mais les exceptions abondent. Par exemple, un homme politique sera le responsable et l'acteur de son discours, mais souvent il n'en sera pas l'auteur, laissant ce rôle à l'un de ses conseillers. De même un bulletin de nouvelles à la télévision (1) est écrit par un ou plusieurs journalistes, (2) se trouve parlé par un présentateur et (3) ) et relève de la responsabilité d'une chaîne. Cette analyse devient remarquablement utile dans l'analyse $\mathrm{du}$ terrorisme. Que se passe t-il, par exemple, quand un kamikaze tient un discours avant de se faire sauter ? Le kamikaze est visiblement l'animateur du discours tenu. En est-il l'auteur ou le responsable?

\section{L.S. : Vous avez parlé de trois auteurs. Qui est le troisième ?}

D.D. : Mon troisième auteur-phare - J.L. Austin - est un philosophe pince-sans-rire, un philosophe du langage quotidien que l'on prend souvent pour un linguiste. De l'œuvre de cet ancien membre des services secrets britanniques, je retiendrai un petit livre intitulé en français Quand dire c'est faire. Austin y questionne le présupposé qui veut que tout énoncé soit une description du monde plutôt qu'un acte. Austin peut faire penser à Stanislavski qui demandait à ses étudiants-acteurs d'entrer en scène à tour de rôle en prononçant l'un après l'autre, les mots «Ce soir! ». Les mots ne changeaient jamais, mais ils devaient constituer un acte chaque fois différent. «Ce soir ! " était ainsi une menace, une promesse, un ordre, un défi, etc. La réflexion d'Austin sur le langage comme force inspire mon travail sur la mise en en visibilité

\section{L.S. : Comment ?}

D.D. : Grâce à J.L. Austin j’ai compris que les 
images en général et celles véhiculées par les médias en particulier (photos de presse, vidéos, images de télévision) ne sont pas simplement des « représentations » de la réalité, fidèles ou infidèles, objectives ou partiales, mais des actes de regard - au sens où Austin parlait d'actes de parole. Au nombre de ces formes de regard qui sont des actes, il y a les regards qui consistent à montrer. En voici un exemple. Dans la Souffrance à Distance, Luc Boltanski évoque les discours de la «compassion » ou de la "dénonciation» par lesquels nous répondons aux images de souffrances lointaines. J'irais un peu au-delà de Boltanski en disant que la compassion et la dénonciation sont effectivement là, mais qu'en fait elles précèdent notre réponse. Elles sont déjà inscrites dans les images de souffrance. Il existe ainsi des images qui compatissent, des images qui dénoncent, et aussi des images qui gênent, comme celles prises par la jeune soldate américaine Sabrina Herman dans la prison d'Abou Ghraib. Ces images gênent car on ne sait si en montrant les humiliations imposées aux prisonniers irakiens, la jeune photographe approuve ou dénonce. Il faudra attendre le scandale provoqué par ces photos l'indignation qui se manifeste lorsque les photos sont reprises et montrées par les grands médias - pour dissiper l'incertitude quant à la nature de l'acte impliqué par chacune des photos. La philosophe Judith Butler, également nourrie de la pensée d'Austin, nous rappelle qu'interpréter une photo, c'est interpréter ce qui est déjà une interprétation. Je dirais que montrer une photo, c'est montrer ce qui est déjà une monstration et que la plupart des images que nous voyons dans les médias sont des palimpsestes. Ces images superposent en effet, plusieurs étages de monstration. Comprendre alors ces images, c'est reconstituer des échafaudages de monstrations superposées, identifier et évaluer les actes que chacune d'entre elles accomplit.

\section{DES "ADVERSAIRES" INTELLECTUELS?}

\section{L.S. : Quels sont ou quels ont été vos adversaires intellectuels?}

D.D. : Comme tout le monde dans nos milieux, j'ai eu des adversaires, des lecteurs hostiles, des auteurs qui ont publiquement attaqué mes positions. Souvent, j'ai répondu à de telles attaques. Parfois ceux qui m'attaquaient sont devenus des amis. Pourtant ce n'est pas d'eux qu'il s'agit ici mais de ceux dont on aurait pu croire qu'ils seraient mes références majeures et qui en fait ne l'ont jamais été. Ceux dont l'œuvre malgré sa proximité avec mon travail ne m'a inspiré ni l'enthousiasme d'un disciple, ni l'énergie d'un iconoclaste. Ceux que j'ai en fait non pas attaqués, mais ignorés.

Il s'agit d'abord de Mac Luhan dont je n'ai jamais aimé les affirmations à l'emporte-pièce, celles à propos desquelles Umberto Eco parlait - cruellement - de « cogito interruptus ». Je ne crois pas du tout que «le medium soit le message » et je préfère à la notion de «Village Global », la description circonstanciée qu'offre Joshua Meyrowitz de la disparition de la notion même de « lieu ».

Vient ensuite Guy Debord et l'équivalence qu'il pose entre spectacle et marchandise. Si cette équivalence permet de reprendre le discours 
marxien, elle dispense malheureusement de toute analyse sur des spectacles particuliers. Le spectacle n'est pas pour Debord une réalité empirique à explorer. C'est un concept explicatif ou le signe d'une déchéance. En un mot je ne m'intéresse pas à Debord, parce que Debord ne s'intéresse pas au spectacle.

Vient enfin celui dont j'aurais dû être vraiment proche : Jean Baudrillard, sémioticien et sociologue. Le Baudrillard qui me concerne n'est pas l'aède post-moderne qui parle de l'Amérique. J'ai passé le tiers de ma vie aux États-Unis et ne reconnais pas ce qu'il décrit. Sans doute suis-je trop « américain » pour voir en Vegas l'emblème du continent. Je suis beaucoup plus sensible à ce qui fait de New York l'une des grandes villes européennes du $\mathrm{XIX}^{\mathrm{e}}$ siècle, marquée par la présence de Vienne, de Londres, de Palerme.

Le Baudrillard qui me concerne est celui de la «Précession des Simulacres», l'analyste de ces zones indécises où l'ordre des images et des simulations devient le fondement de la réalité collective. Ce Baudrillard-là est éblouissant, mais à tous les sens du terme : il illumine. Il aveugle. Baudrillard décrit mieux que personne un phénomène important et troublant. Baudrillard déconcerte parce que, porté par des enthousiasmes successifs, il joue un peu capricieusement à convoquer, puis à révoquer la réalité. Disparue avec l'ère des simulacres, elle serait de retour avec le 11 septembre. Il est parfois difficile de voir où commencent et où s'arrêtent les simulacres. Il y a longtemps me semble-t-il que des ordres fondés sur la représentation fondent les réalités sociales. N'est-ce pas de cela que parlait déjà Rousseau, dans la Lettre sur les Spectacles? Y a-t-il des sociétés sans « Semiosis »?

Peut-être mon problème vis-à-vis des trois auteurs que je viens de citer tient à ce que indépendamment de ses qualités rhapsodiques, une théorie est avant tout pour moi un instrument qui permet de décrire, et dont les ambitions sont donc suffisamment restreintes pour pouvoir être « falsifiables ».

\section{TERRORISME, ISLAMISME}

\section{L.S. : En quoi l'islamisme est-il un médium ?}

D.D. : En termes littéraux, il est difficile de concevoir l'islamisme comme un médium. La notion de médium s'oppose à celle de message ou de contenu et les islamismes (puisqu'il y en a plusieurs ) se définissent précisément par la nature des messages qu'ils énoncent. Pour pouvoir être des "médias" il faudrait, me semblet-il, que les islamismes ne se confondent pas avec des contenus spécifiques; qu'ils véhiculent un spectre de messages suffisamment large pour ne pas s'identifier avec certains d'entre eux.

Plutôt que de « médium » peut-être pourraiton alors parler de « langage ». Les islamismes pourraient alors être conçus, non seulement comme des contenus doctrinaux, mais comme un moyen d'exprimer le mal-être de populations humiliées, leur malheur, leur désir de dignité. Ils seraient des «symptômes », des épiphénomènes.

Cette approche «explicative» présente pourtant plusieurs inconvénients. La posture 
« explicative » amène souvent à ne pas vraiment écouter le discours qu'on explique et donc à en sous-évaluer la portée. Inversement, le fait qu'une doctrine soit vue comme l'expression indirecte d'un malheur amène à ne plus voir ce qu'il peut y avoir d'inacceptable dans certains aspects de la doctrine, et à ne plus voir que le malheur. Si je devais faire une interprétation « communicationnelle» des islamismes, elle ne consisterait donc ni à en faire des médias, ni même à en faire des « langages ».

Je choisirais plutôt de situer les islamismes dans la perspective représentée par l'historienne du protestantisme Elizabeth Eisenstein : celle d'une anthropologie de la lecture. Quelles questions soulèverait alors une telle perspective? Sur les milliards de musulmans que compte la planète, il existe plusieurs milliards qui ne savent que quelques mots d'arabe. Quant aux populations qui parlent et comprennent l'arabe, toutes ne comprennent pas celui du Coran. Enfin de ceux qui parlent ou comprennent un arabe relativement proche de celui du Coran, une vaste proportion ne sait pas lire (ou en tout cas, ne sait pas lire l'arabe). Faute d'être lu, analysé, le Coran devient alors mémorisé, canonisé. Faute de pouvoir être interprété, reformulé, paraphrasé, le Coran se fige dans une version autoritaire,. Faute d'être porté par un médium autre que la mémoire, le texte sacré est simplifié. Une orthodoxie inquiète s'empare de l'exégèse et prône des interprétations littéralistes. Face à l'islam des lettrés se constitue alors un islam $\mathrm{du}$ « par cœur ». Je me demande alors jusqu'à quel point les islamismes ne sont pas aussi la conséquence politique d'un certain rapport à la lecture.

\section{L.S. : En quoi le terrorisme est-il un médium ?}

D.D. : La plupart des actes terroristes s'adressent à une opinion publique et ont l'ambition d'agir sur cette opinion, que ce soit celle des pays cibles dont il s'agit d'intimider ou de convaincre les populations " par-dessus la tête» de leurs dirigeants; que ce soit celle des populations « représentées » par les terroristes dont il s'agit alors de s'assurer rétrospectivement le soutien; que ce soit celle des terroristes potentiels chez lesquels il importe de susciter des vocations; que ce soit enfin celle d'autres groupes terroristes, dont il s'agit d'obtenir la coopération ou le respect.

Cet aspect communicationnel suffit-il pour dire que le terrorisme est un médium? Non. Mais il me semble néanmoins que le terrorisme est un médium. Partons d'une constatation à la fois triviale et surexploitée. Il existe non pas un, mais des terrorismes. Ceux-ci différent par leurs contextes historiques, par leurs contenus, par leurs méthodes, par leur relation à l'éthique, par leurs idéologies. Cette diversité est telle que l'on finit par dire que ces « terrorismes" n'ont rien de commun sinon une désignation qui les marque du sceau de l'infamie.

Réveillons-nous disent alors certains. Frottonsnous les yeux. Nous verrons ainsi que parler de « terrorisme » c'est procéder à un amalgame ou à une hypostase; c'est valider comme réalité ce qui n'existerait qu'au niveau des mots. Les terroristes sont des "guérilleros» calomniés. Qui veut discréditer son opposant l'accuse de terrorisme. Supprimons alors le mot terrorisme. 
Le terrorisme n'existe pas. Ne parlons plus de terrorisme. Ne parlons plus que de militants.

Outre le fait qu'elle consiste à fonder une «novlangue» riche en énoncés surréalistes («Ce matin, des "militants" ont tué quarante personnes dans un autobus ») la suppression du mot « terrorisme » me semble absurde. Il est facile de remarquer qu'il existe des « militants » qui ne tuent personne. Ces militants défilent, distribuent des tracts, vendent des journaux, collent des affiches ou proclament l'invincibilité $\mathrm{du}$ peuple. Certes les terroristes sont des militants, mais tous les militants ne sont pas des terroristes. Pour ceux qui pensent que les vies humaines ont une importance, la distinction compte.

Mais je note un second paralogisme. Affirmer qu'une réalité sociale n'existerait pas dès lors qu'elle est soumise à des variations serait aussi absurde que de dire que la notion d'assassinat n'existe pas puisqu'il y a des assassinats passionnels, des assassinats politiques et des assassinats crapuleux. Où veux-je en venir?

À ceci. Pour se désigner eux-mêmes, les terroristes ne parlent jamais de terrorisme. Ils préfèrent utiliser d'autres mots, se focaliser sur les fins qu'ils se donnent plutôt que sur les moyens qu'ils utilisent. En revanche, pour ceux qui, comme moi, persistent à utiliser le mot « terrorisme » le terrorisme se définit, non pas par des buts (variables), mais par la nature des moyens employés. Quels que soient les messages véhiculés et les bénéficiaires de ces messages, la communication que le terrorisme instaure passe toujours par une certaine forme de violence ; par une violence à la fois spectaculaire et limitée. C'est cette violence spectaculaire et limitée qui définit la communication terroriste, et non pas des contenus dont il importe peu qu'ils soient admirables ou abjects.

On comprend alors pourquoi la multiplicité des terrorismes ne mène nullement à conclure que le terrorisme n'existe pas. Elle signifie simplement que le terrorisme est un médium et que, comme tout médium, il se prête à plusieurs sortes de contenus.

Le fait de voir le terrorisme comme un médium répond simultanément à un second argument qui est celui du «n'a pas le choix ». Ceux qui pratiquent le terrorisme «n'auraient pas le choix », nous dit-on, puisqu'il s'agirait du seul moyen possible pour certains groupes humains de se faire entendre. C'est faux. Bien sûr qu'ils ont le choix. D'autres médias peuvent véhiculer les mêmes messages. La manifestation publique en est un. La résistance non-violente en est un autre. L'auto-immolation en est un troisième. Par exemple, celle d'un jeune marchand de légumes tunisien a soulevé plus d'émotion dans le monde que les dizaines d'actes terroristes survenus la même semaine. Le terrorisme est donc un médium. Mais, dans les situations d'oppression, il n'est ni le seul des médias disponibles, ni même le plus efficace. 
$R \cdot E \cdot F \cdot E \cdot R \cdot E \cdot N \cdot C \cdot E \cdot S$

John Langshaw AUSTIN. Quand dire, c'est faire, Seuil, Points Essais, 1991.

Roland BARTHES. « L'écriture de l'événement » in Communications, 12, 1968.

Roland BARTHES. « Le discours de l'Histoire » in Le bruissement de la langue, Essais critiques IV, Seuil, Points Essais, 1993.

Roland BARTHES. Mythologies, Seuil, Points Essais, 1970.

Luc BOLTANSKI. Souffrance à Distance, Gallimard, Folio essais, 2007.

Daniel DAYAN et Elihu KATZ. La Télévision cérémonielle, Collection La Politique éclatée, Puf, 1996.

Philip SCHLESINGER. Putting "reality" together : $B B C$ news, Routledge, Revised edition, 1987. 